\title{
Aerodynamic and Propulsion Assisted Maneuvering for Orbital Transfer Vehicles
}

\author{
Patrick R. Jolley ${ }^{*}$ and Stephen A. Whitmore ${ }^{\dagger}$ \\ Utah State University, Logan, Utah, 84322
}

\begin{abstract}
The ability to perform rapid orbital transfers will provide the ability for unpredictable reconnaissance or timely rescue missions. This paper examines the means and ways of enabling responsive space through propulsive and aero-assisted maneuvers. A conical hypersonic waverider was designed and its aerodynamic database was generated using hypersonic incidence angle analysis tools with a viscous skin-drag correction. Sample trajectories were analyzed for heating levels. Transpirational cooling along the leading edge or the use of ultra-high temperature ceramics are needed to control heating. A throttled engine regulator control was built to provide sufficient energy to maintain the orbit. The regulator control law is derived and analyzed for performance ability. The orbital transfer trajectories were analyzed using an interactive simulation tool. Results have confirmed previous research that aero-assisted maneuvers are more efficient than purely propulsive maneuvers alone for executing synergetic plane changes.
\end{abstract}

\section{INTRODUCTION}

CURRENTLY, the state of all LEO objects are in known orbits, whose parameters make it very easy to predict their future place and time through orbital ephemeris. The military assets in space are also known and tracked for known coverage times. Reconnaissance satellites cannot easily be deployed for rapid or sudden changes in events. Orbit phasing may take several hours or days before an acceptable overhead pass produces good results. Obtaining the ability to perform radical orbital changes and quick responsive launches will eliminate the known overhead window and replace it with a when-needed window.

The U.S. Government has expressed a need to create an operationally responsive space. The public sector, in addition, would definitely benefit from more responsive space based assets. The vision for responsive space is to create or build the ability for rapid response capability. This means the ability to place an asset into a desired orbit for small payloads within hours of the projected need. This ability would grow to include larger payloads for a more adaptive and agile operating environment. The idea behind operational responsive space is to achieve the ability to place an asset rapidly into a desired Earth centered orbit and do so economically. The ability to repair, rescue, rendezvous, and recycle quickly are the advantages of responsive space. Furthermore, the very nature of the way we use space will

\footnotetext{
Graduate Research Assistant, Mechanical \& Aerospace Engineering, 4130 Old Main Hill, Student Member AIAA

$\dagger$ Assistant Professor, Mechanical \& Aerospace Engineering Dept., 4130 Old Main Hill, Associate Fellow AIAA.
}

be changing as a result of NASA's plan to Return to the Moon. There will be several launches into LEO just to place the required assets in place to facilitate a trip to the Moon. The commercial sector is growing and is steadily pursuing the dream of building a space hotel. With the addition of more valuable assets going into LEO the need for the ability to reach them quickly is also rising. Aero assist maneuvers will change the way that we use LEO and the assets that we place there. However, it is important to consider why aero assist maneuvers are so important for a spacecraft to provide responsive space. High maneuverability in space comes at a great $\operatorname{cost}^{1}$. The $\Delta V$ required to execute a plane change of significant value is extremely large.

$$
\Delta V=2 V_{i} \sin (\theta / 2)
$$

\section{Equation 1}

The equation above illustrates the fact that when considering that a plane change the required $\Delta V$ is roughly proportional to the initial velocity. This amount of $\Delta V$ is prohibitive because of the amount of extra fuel that would also be required to be launched into orbit. It is for this reason that spacecraft do not normally execute plane changes by propulsive means. Re-tasking a reconnaissance satellite by means of its onboard propellant supply would essentially reduce the effective lifetime by $50 \%$ or more. The only other option to reducing the cost of an orbital plane change is to increase the Isp of the propellant being used. Although the $\Delta V$ requirement remains the same the amount of propellant would be significantly reduced. Some alternatives to the mainstay of propulsion systems that offer a high Isp are electric propulsion systems. The only drawback is their very low thrust level output. This disadvantage disqualifies them for performing in a responsive space mission role because of the long duration needed to execute their maneuvers. Another option for creating a responsive space lies in how assets are placed into orbit. The available alternatives and options for placing a satellite into an Earth centered orbit are discussed below.

\section{ENABLING RESPONSIVE SPACE}

\section{A. Conventional Launch Platforms}

Conventional launch platforms require long lead times and are constrained to a minimal number of launch sites thereby reducing further their ability to timely place a satellite into a desired orbit. The Orbital Pegasus launch vehicle is a reliable air launched system. The Pegasus satellite launch vehicle also has the disadvantage of lead time of several days and the requirement of its dedicated aircraft.

The USAF recently conducted a feasibility study ${ }^{2}$ to determine alternatives to meet the responsive requirement. This study researched the idea of using an F-15 to carry a microsatellite launch vehicle. This program appeared very attractive because it used an operationally benign aircraft already in service around the world. However, the program eventually failed to meet all of the design requirements. 


\section{B. Orbit Modification}

The current ability of a satellite to change its orbit depends upon its propulsion system. Satellites require a small propulsion system to maintain a specified orbit. However, they are often times equipped with a second, larger system, in terms of thrust, to perform maneuvers specific to their missions. These include orbit circularization, co-planar transfers, and orbital plane changes.

If the satellite happens to be in the desired orbit a phasing maneuver can be executed to simply shift the position forwards or back as needed. Depending upon the positional change required and the available propellant budget, a phasing maneuver can be carried out over a period of a few days or can be achieved within just a few orbits.

Most satellites do not arrive in exactly the specified orbit or because of the launch site it is simply impossible to place it there. Out of plane changes must then be executed to remedy this. This maneuver is the most prohibitive to conduct because of its high cost in propellant consumption. Satellite lifetime is coupled closely to the remaining available propellant.

The second alternative to space based maneuvers includes taking advantage of the atmosphere below rather than avoiding it in the above case. The maneuver is known commonly as a synergetic plane change and is accomplished through a combination of aerodynamic and propulsive forces. This method requires that the satellite be packaged into a vehicle with aerodynamic qualities. Moreover, it has been suggested by Walberg $^{3}$ that the lift to drag ratio must be greater than one and that the required plane change be greater than 15 degrees before a synergetic plane change becomes more efficient than a conventional propulsive burn alone.

The ability to execute a synergetic plane change is a valuable asset currently not available on satellites or space vehicles. If a spacecraft could perform a synergetic plane change as described it would be able to perform a variety of operations. An important ability would provide the government with unpredictable reconnaissance. The current reconnaissance assets of the U.S. Government are in known locations with known field of view times. Enabling a spacecraft with synergetic plane changes means that an asset could be moved to cover a new area at will and at times unknown to hostile forces. Beyond the reconnaissance application is the ability of a highly agile spacecraft to perform multiple objectives without the need for multiple surface launches. These objectives could include rescue, repair, return, or recycling.

The emphasis of this paper will be to analyze more in depth the option of synergetic plane changes to enable responsive space. This method has been studied in theory before. Nevertheless this paper will explore the use of a three degrees of freedom simulator to assess the real abilities and real deficiencies of this method for providing a means to achieving responsive space.

The sequence of operations to perform a synergetic plane change is as follows. Ideally, the spacecraft will lower its orbit through a retrograde burn until it interfaces with the atmosphere, at which point it will bank in order to roll the lift vector in a lateral direction.

The Space Shuttle routinely uses this method to modulate its reentry. In fact, the cross range ability of reentry vehicles is tied directly to their ratio of lift-to-drag ${ }^{4}$. When performing a plane change, however, the spacecraft desires to maintain its orbital energy. Therefore, the vehicle must roll upside down and keep its lift vector pointed towards the center of the Earth to counteract the centrifugal forces. After achieving the desired plane change the spacecraft will then engage its propulsion system again to boost back up into its original orbit.

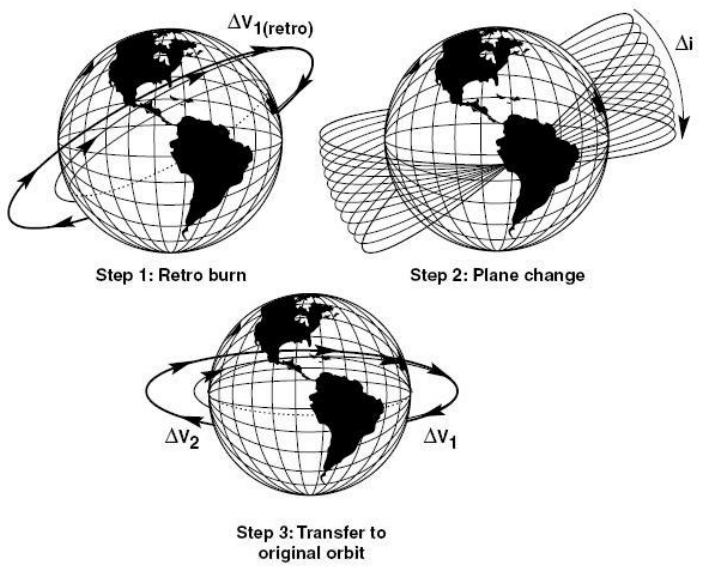

Figure 1- The Three Steps for Aero-Assist

\section{TECHNOLOGY REQUIREMENTS}

\section{High L/D Hypersonic Airframe}

A plane-change aerodynamic maneuver is most efficiently performed when the vehicle operates very near the the maximum hypersonic L/D ratio ${ }^{5}$. For hypersonic conditions, high L/D configurations have long slender shapes and sharp leading edges that minimize the supersonic wave drag of the wing. The blunt shapes of the lifting bodies necessary to produce lift at low speeds and to dissipate the heat of reentry do not allow for good hypersonic L/D performance.

High L/D performance directly relates to several areas important to performing an aero-assisted maneuver but will be explained from the re-entry point of view. For landing at sites at latitudes higher than the orbital inclination the spacecraft must have the ability to maneuver in a direction that is out of the orbital plane of inclination. The better means of reaching landing sites at latitudes higher than the orbital inclination is to use aerodynamics during the reentry. The lift vector is rolled in a direction transverse to the flight path and effectively increases the orbital inclination. The critical factor for achieving high cross range is the maximum lift-to-drag ratio of the vehicle. The available down range is also related to the $\mathrm{L} / \mathrm{D}$ of the vehicle.

The body shape that most favors a higher $\mathrm{L} / \mathrm{D}$ ratio also exhibits two other disadvantages: high heating and poor volumetric efficiency.

\section{Sharp Leading Edges Thermal Management}

To reduce wave drag the leading edges of wings and bodies must be sharpened. The consequence ${ }^{6}$ is that the stagnation heating from hypersonic flow is inversely proportional to the square root of the radius of the surface. Hence, the larger the radius the less heating that will occur. For example, the Space Shuttle has blunt leading edges to help dissipate the heat generated back into the flow. In addition, a blunt leading edge will cause the shockwave to become detached from the vehicle and thereby reduce the hypersonic L/D considerably from the loss of compression lift. Without the ability of sharp leading edges the aero-assist option is not as favorable an option. 


\section{E. Restartable, Variable Throttle Engines}

Aero-assist maneuvers require the ability to have real time feedback to ensure that the spacecraft will have enough energy to compensate for drag losses encountered in the atmosphere. The aerodynamic pass of the spacecraft will not be without uncertainties. Therefore, a simple prescribed amount of thrust for each pass cannot be calculated exactly beforehand. Additionally, each successive aero pass will require a different level of thrust than the time before. For these reasons it is necessary that the spacecraft be equipped with a variable thrust engine, or an engine that can be throttled to give the needed levels of operation. One example of a throttled engine successfully used before is found in the Apollo program. The lunar ascent module carried an engine that could be throttled to provide the astronauts the level of thrust required for their mission.

The propulsion system must also be capable of multiple restarts due to the number of aero passes required to complete the desired plane change.

\section{THE WAVERIDER VEHICLE}

One candidate vehicle that exhibits the necessary qualities as set forth is commonly known as the waverider. Waveriders are an area of unexpected related research that has begun to be pursued in space based aero assist maneuvers. Why the exotic waverider vehicle? The answer lies within the vehicle's proposed capabilities and its current limitations. But first, what is a waverider? John D. Anderson ${ }^{7}$ explains it this way. "A waverider is a supersonic or hypersonic vehicle that has an attached shock wave all along its leading edge at design point conditions."

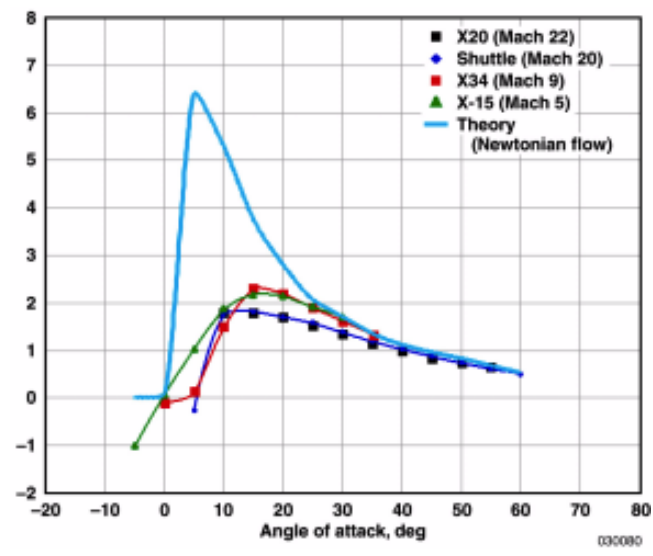

Figure 2- L/D for a variety of vehicles.

The attached shock wave and all of its advantages thereby generated are available at "design point conditions." Unfortunately, as the vehicle Mach number changes, so does the local shock wave incidence angle interactions with the vehicle. The performance of the vehicle is maximized at only one design point as is often the case and yet not entirely realized under all conditions. Figure 2 above is a plot of hypersonic L/D ratios for four different wing-body re-entry configurations compared to the theoretical predictions for a flat plate using modified Newtonian flow.

There are several things that are important to understand from the definition as given by Dr. Anderson. First, waveriders are designed for hypersonic flight. When a vehicle has an attached shock wave it gains an aerodynamic advantage in that the high pressure field is contained under the vehicle. This equates to a higher lift force generated upon the vehicle. Waveriders are the only vehicles that can generate high lift-to-drag (L/D) ratios that are needed to perform maneuvers such as trajectory modification. Several researchers at the University of Maryland ${ }^{8,9}$ have investigated possible waverider configurations that are optimized for high $\mathrm{L} / \mathrm{D}$ ratios.

Jon A. Sims ${ }^{10}$ and a team of researchers from the Jet Propulsion Laboratory have explored the idea of augmenting gravity-assist with aero-assist techniques to aid in exploring the outer planets. Using waveriders to perform aero-assist maneuvers can significantly reduce the overall mission travel time by a factor of two to three times as can be achieved under normal flight trajectories ${ }^{11}$. Waveriders are the only vehicle capable of performing these kinds of maneuvers in hypersonic flight.

A waverider vehicle was therefore chose as the vehicle of choice for this study into aero-assist maneuvers. The waverider that was chosen for this study is a conical power law waverider with a power law exponent of approximately 0.74 . The corresponding body dimensions are an 11 meter length and a 2.76 meter half width. The cone was also sliced longitudinally by a depth of 0.6 meters to provide for a rounded nose instead of a sharp tipped nose. An estimation of the vehicle's center of gravity was made by assuming the nose of the vehicle would be composed of a heavy heat sink much like that of NASA's X-43 vehicle.

\section{WAVERIDER VEHICLE AERODYNAMICS}

Although waveriders have been proposed for several interplanetary missions, they have not been fully examined for their flight worthiness. The waverider suggested in this study is therefore outfitted with several different flight control surfaces. The surfaces included are similar to what are expected on flight vehicles; namely, body flaps, a rudder, and wings with flaps. The effectiveness of each of these surfaces in maintaining trim flight will also be examined. Consequently, because this waverider will exhibit control surfaces and minimally blunted edges its peak performance will be considerably less than what can be analytically predicted. However, the point of this exercise is to produce a realistically capable flying waverider. This section, therefore, presents a summary of the aerodynamic databases that were calculated for the different flight configurations. The figure below illustrates the model that was created to generate an aerodynamic database for a conical power law waverider.

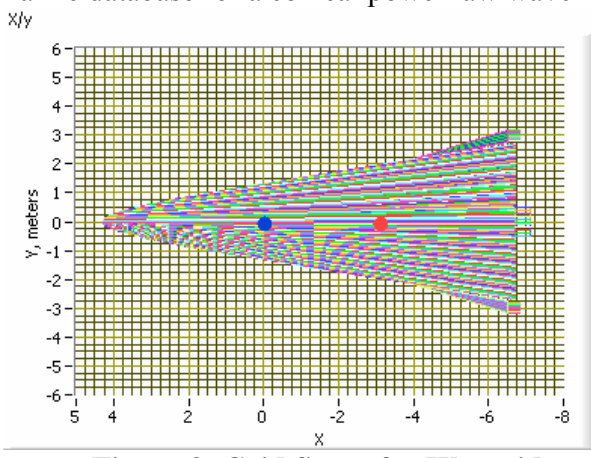

Figure 3- Grid Setup for Waverider

\section{F. Aerodynamic Modeling Techniques}

The aerodynamic database was generated using simple hypersonic incidence angle analysis tools. ${ }^{12}$ The type of solution technique used for a particular computational grid cell depends on the local incidence angle, and whether the local surface is spherical, conical, or flat. 
For conical surfaces, the "tangent-cone" method was used to predict the windward surface pressure. The tangent cone method was implemented in the analysis code by integrating the TaylorMaccoll ${ }^{13,14}$ equations.

For flat surfaces like the trim flaps oblique shock wave theory (Ref. 7) was used to predict the windward surface pressures.

Because of hypersonic heating across the bow shock wave, $\gamma$ is not 1.4 and changes across the bow shock wave. An averaged value for $\gamma$ across the shockwave is calculated using Eckert's ${ }^{15}$ empirical reference temperature

$$
T_{r e f}=T_{\infty}+\frac{1}{2}\left(T_{a_{w a l l}}-T_{\infty}\right)+0.22\left(T_{02}-T_{\infty}\right)
$$

\section{Equation 2}

In Eq. (2) $T_{\text {ref }}$ is the reference temperature, $T a_{\text {wall }}$ is the adiabatic wall temperature, $T_{\infty}$ is the freestream temperature, and $\mathrm{TO}_{2}$ is the stagnation temperature behind the shock wave.

For a given Mach number the temperature equations are solved iteratively. For each of the iterations the reference temperature and stagnation pressure behind the shock wave are used to look up the gas properties $\left(\mu, C_{p}, \kappa, \gamma\right)$ using real gas tables for air. ${ }^{16}$

Once the surface pressure distribution on the spherical, conical, and flat sections of the waverider grid were computed, the forces at each cell were resolved into the axial, normal, and lateral directions, and summed, and non-dimensionalized to give the normal $\left(\mathrm{C}_{\mathrm{N}}\right)$, axial $\left(\mathrm{C}_{\mathrm{A}}\right)$, and lateral $\left(\mathrm{C}_{\mathrm{Y}}\right)$ force coefficients. The normal and axial force coefficients are related to the lift and drag coefficients by

$$
\begin{aligned}
& C_{L}=C_{N} \cos (\alpha)-C_{A} \sin (\alpha) \\
& C_{D}=C_{N} \sin (\alpha)+C_{A} \cos (\alpha)
\end{aligned}
$$

\section{Equation 3}

The approximate aerodynamic (AC) center of the waverider model was approximated by the integrated center of pressure of the vehicle at zero angle-of-attack. All aerodynamic moments were calculated by integrating the resolved normal, axial, and lateral pressure forces at each cell with the appropriate moment arm with respect to the approximate aerodynamic center.

\section{G. Drag Corrections}

The effects of skin friction were modeled using a simple flatplate approximation with a compressibility correction. In this model the Karman-Schoenherr Equation for incompressible skin friction ${ }^{17,18}$ is solved numerically for the longitudinally averaged skin friction coefficient, $C_{F}$, based on Reynolds number, $\mathrm{Re}_{\mathrm{L}}$, for the total body length with the density and viscosity evaluated at reference conditions within the boundary layer ${ }^{19,20}$.

The leading edge drag coefficient due to a blunted leading edge was also calculated and added to the overall drag coefficient.

\section{H. Trim Flight}

A simple Monte-Carlo type analysis was performed once the control surfaces were defined and integrated into the aerodynamic routine to determine each of the aerodynamic coefficients. This type of analysis was akin to flying the waverider through all possible flight scenarios through the adjustment of altitude, Mach number, angle of attack, and beta or sideslip angle. Additionally, the control surfaces were deflected to determine the amount of control authority they had and to determine if it was sufficient to control the vehicle.
The first requirement on the vehicle is that based on angle of attack the body flaps could provide a pitching moment of zero. Because of the configuration of the of the body flaps they will ultimately become ineffective after an angle of attack of four degrees. After four degrees the body itself produces a small negative pitching moment that is the limit of the body flaps. As well, the body flaps are completely ineffective when deployed to a negative amount which means they are being stowed because they are hidden in the vacuum area created by the base of the vehicle. Flight at different altitudes and Mach numbers has no appreciable effect on the pitch coefficient when being trimmed with the body flaps.

By a similar manner, the trim conditions for the rudder and wings were found by iterating through different flight conditions. The results from one of these runs are presented below. Information found from these types of analysis is important in understanding whether the vehicle can be flown in trim conditions through the use of its control surfaces. If excessive control authority is required it can be a sign that the control surfaces are too small. Figure 4 is important to show that although the vehicle is unstable in yaw, due to its natural construction, there is sufficient control authority to provide for trim flight. The vehicle is stable in roll and also can be trimmed.
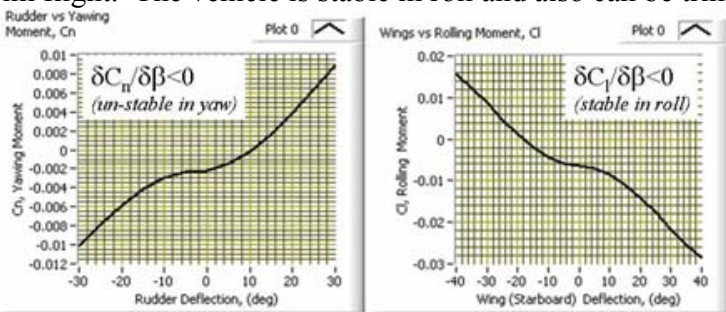

Figure 4- Trim Deflection for Rudder and Wings

Figure 5 is a plot of the data for $\mathrm{L} / \mathrm{D}$ for a symmetric control surface configuration generated at an altitude of $60 \mathrm{~km}$ and zero sideslip angle at four different Mach numbers. The peak of the L/D plots, near two degrees, shifts slightly towards an angle of attack of one degree with the addition of sideslip angle to either side.

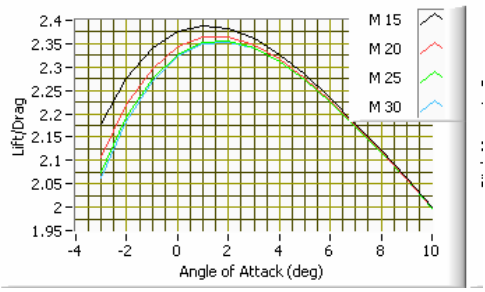

Figure 5- L/D and $\mathrm{Cm}$ for $60 \mathrm{~km}$ and 0 beta

Additional plots were generated to ensure that the yawing moment and rolling moments were of the correct sign to ensure stability. A negative rolling moment is required to recover from a positive disturbance in bank angle. A positive yawing moment is required for static stability for the same conditions.

The aerodynamic databases can be easily and quickly regenerated for different body shapes and control surfaces for different configurations.

\section{PROPULSION}

\section{Orbital Decay}

To obtain an aero-assisted plane change in a strategically meaningful time the spacecraft must operate at altitude levels that are dangerous to maintaining its orbit. The lower the spacecraft 
dips into the atmosphere the more aerodynamic performance will be extracted. Conversely, more energy will be lost to drag in this case. Variable flight conditions are another concern that must be considered in addition to the need to maintain the orbital energy sufficient that the orbit does not collapse and require the spacecraft to reenter. Each aero pass will be significantly different than the one previous because of a different atmospheric entry angle and location and duration of flight. A simple planned impulse burn would be difficult to predict and even a small miscalculation would be catastrophic. Therefore, the focus is on a propulsion system that can deliver a variable impulse bit. The delivery of variable thrust from a rocket engine is known as "throttling."

By modulating the engine thrust near the orbit's perigee the semi-major axis, and apogee, can be maintained. This technique is required because the perigee is extremely low in order to obtain a benefit from flying through the atmosphere. The lower the perigee the more dynamic pressure is available for aerodynamic purposes, but it also means more energy is lost due to drag. When energy is lost to drag or a propulsive maneuver at perigee the result is to lower the apogee of the orbit while the perigee remains relatively constant. Eventually, when enough energy has been lost the orbit will collapse.

Figure 6 shows the approximate orbital decay rate of a notional vehicle in low earth orbit. The plot displays a very distinct "knee" in the curve where the orbit begins to decay catastrophically. The key to the aero-assist maneuver is to generate lift while flying through the atmosphere and still maintain a sufficiently high enough apogee to stay above the point of orbit collapse. If the apogee is kept above the knee in the curve there will be enough energy to perform at least one more orbit.

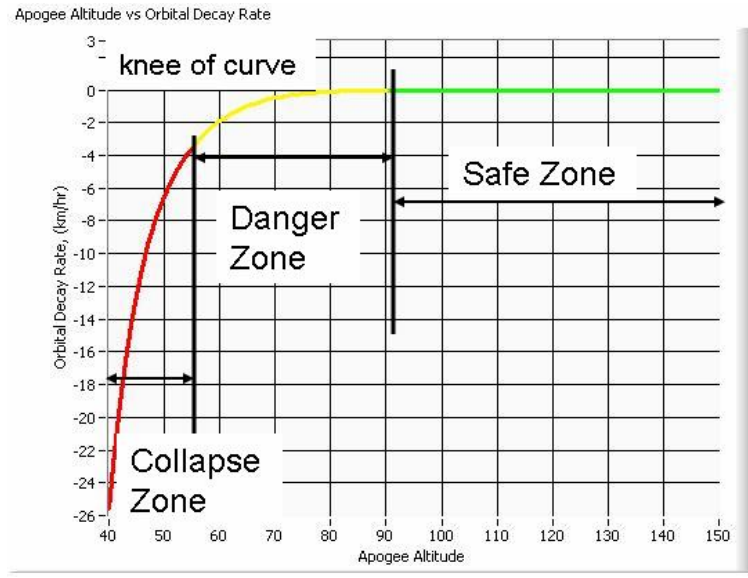

Figure 6- Curve of Decaying Orbital Energy

The ideal location for the vehicle to operate becomes the knee of the curve. However, operating near the "knee" of the curve has obvious risks. The atmosphere is highly rarefied and the vehicle's drag characteristics can only be predicted. Only a small variation in the aerodynamics could send the spacecraft "over the cliff."

\section{J. Propellant Choice}

Because the engine required for aero-assist will need to be restarted several times a solid propellant motor will not work. Liquid rocket engines are very suitable to restarts and also provide the most opportunity for throttling. Several different liquid propellant choices were examined as options for this mission using NASA's Chemical Equilibrium with Applications code. The code is used to calculate chemical equilibrium compositions and properties of complex mixtures including applications for theoretical rocket performance calculations ${ }^{21}$.

Most propellant budgets are limited by mass available and therefore mission planners choose the propellant combination that delivers the most Isp. However, the waverider vehicle will probably be more constrained by available volume than mass because of the slender body does not have as a high of a packaging factor. Consequently, it is also important to consider the densities of the available fuels to determine which fuels can be packaged the smallest. Example cases were created to analyze these differences. The sample cases were an arbitrarily chosen available propellant budget mass limited by $10,000 \mathrm{~kg}$ or volume limited by $10 \mathrm{~m}^{3}$.

It is obvious that the propellant choices with the highest Isp values have LOX as the oxidizer. These results are the expected outcomes when considering a propulsion system limited by mass. For the given amount of mass available the higher the Isp value the more $\Delta V$ can be extracted. However, when considering volume, Fig. 7 shows that the propellant combinations which have the highest Isp unfortunately also have the highest volume requirements.

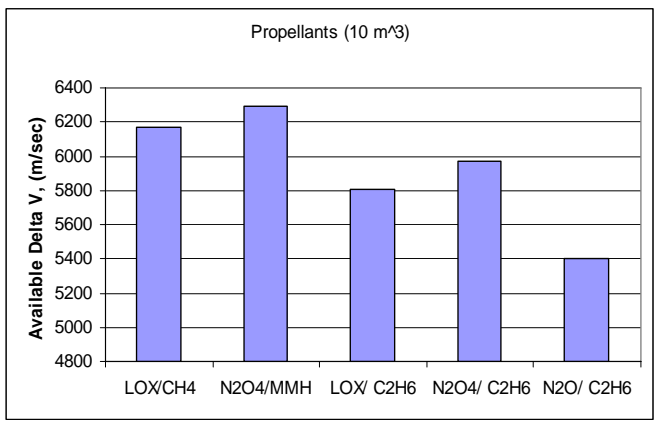

Figure 7- Propellants vs Delta V by Volume

The choice for $\mathrm{N}_{2} \mathrm{O}_{4} / \mathrm{MMH}$ becomes optimal when the spacecraft is volume limited rather than mass limited as is the case for the waverider because of its slender body. The slender body lends itself well to the packaging of cylindrical propellant tanks.

$\mathrm{N}_{2} \mathrm{O}_{4} / \mathrm{MMH}$ has the added benefit of storability and an extensive heritage with spacecraft propulsion systems. Using LOX as one of the propellant choices either reduces the available service time due to boil off, or forces the vehicle to carry a cooling system for the propellant. $\mathrm{N}_{2} \mathrm{O}_{4} / \mathrm{MMH}$ is hypergolic which means that when the two parts come together an immediate reaction occurs. This reduces complexity in the system by eliminating the need for an ignition system and removes a possible failure source.

\section{K. Closed Loop Throttle Model}

The design approach for the orbital regulator is to modulate thrust in such a manner that original orbital energy is maintained. A block diagram that illustrates an overview of the close loop throttle model is shown below in Fig 8. Each block in the diagram will be discussed in the succeeding sections.

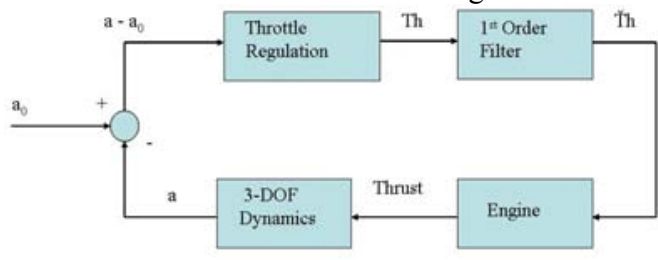

Figure 8- Engine Closed Loop Block Diagram 
The orbital regulator state equation is derived and will be presented below. The orbital decay rate due to non-conservative forces is

$$
\varepsilon=\frac{\mu}{2 a^{2}} a=\frac{\bar{F}_{\text {non-conservative }} \cdot \bar{V}}{m}
$$

\section{Equation 4}

Equation 4 demonstrates that the orbital energy decay is proportional to the rate of decay of the semi-major axis of the orbit. For regulator design, it is convenient to reformulate the orbital decay equation in terms of a "differential energy equation", that is: $a-a_{o}$. The purpose of the orbit regulator is to drive the magnitude of $a-a_{o}$ is small.

$$
\frac{d}{d t}\left[a-a_{0}\right] \approx 2 \frac{\left(a-a_{0}\right)^{2}}{\mu} \frac{\bar{F}_{\text {non-conservative }} \cdot \bar{V}}{m}
$$

\section{Equation 5}

A Taylor's series is used to achieve a reasonable derivative that can be applied numerically by expanding the energy equation about $a_{0}$. Using the definition of $\mathrm{x}=\mathrm{a}-\mathrm{a}_{0}$ allows Eq. (5) to be rewritten as

$$
\frac{d x}{d t}=4 \frac{a_{0}}{v} \times \frac{\bar{F}_{\text {non-conservative }} \cdot \bar{V}}{m}
$$

\section{Equation 6}

Thrust will oppose the direction of $\mathrm{dx} / \mathrm{dt}$ therefore if the orbit is shrinking then energy must be added. In Equation 6, the nonconservative force acting on the spacecraft is primarily atmospheric drag. The engine will be used to replace the energy removed by the drag forces experienced while performing an aero pass.

$$
\bar{F}_{\text {thrust }}=-\bar{F}_{\text {non-conservative }}
$$

\section{Equation 7}

The engine thrust, $F_{\text {thrust }}$, is proportional to the throttle setting; and the commanded engine thrust can only be within the limits of the engine.

The inner product of the nominal thrust and velocity vector is

$$
\bar{T}_{\text {nom }} \cdot \bar{V}=\left|\bar{T}_{\text {nom }}\right|\left[V_{r} \sin \left(\theta+V_{v} \cos (\theta)\right)\right]
$$

\section{Equation 8}

In Eq. (8), $V_{r}$ is the radial (vertical) velocity of the spacecraft, $V_{v}$ is the circumferential (horizontal) velocity, and $\theta$ is the pitch angle of the spacecraft. The final form of the state equation for the orbital regulator is given below.

$$
\frac{d}{d t} x=-2 \frac{x^{2}}{\mu}\left[\frac{\left|\bar{T}_{\text {nот }}\right|\left[V_{r} \sin \left(\theta+V_{v} \cos (\theta)\right)\right]}{m}\right] T
$$

\section{Equation 9}

\section{Optimal Control Law}

As discussed earlier, the goal of the orbital regulator is to drive the state variable $x$ to zero. Since $x=a-a_{0}$, this control action would have the effect of stabilizing the orbit at the original energy level. In Eq. (9), the differential energy $x$ is the state variable, and throttle $T$ is the control variable. Conceptually, the pitch angle, $\theta$, could also be modulated as a control variable. However, simulation results quickly demonstrated that the optimal pitch angle is the value that gives the maximum $\mathrm{L} / \mathrm{D}$ ratio for the spacecraft. Thus, including pitch angle in the feedback loop adds unnecessary complexity to the control laws.
Following the procedure for laid-down by the Calculus of Variations $^{22}$ the first step towards optimality is to define a scalar cost functional which measures the performance of the regulator. The form of the performance index is

$$
J(x, T)=\frac{1}{2} \int_{0}^{t}\left[q_{1} x^{2}+q_{2} T^{2}\right] d t
$$

\section{Equation 10}

The goal of the regulator is to minimize $J$ subject to the constraints of the state equation. The regulator seeks to drive the state variable $x$ to zero; however, unnecessary engine throttle activity, wastes valuable fuel, and is also penalized in the regulator. The gain parameters in Eq. (10), $q 1$ and $q 2$, weight the relative cost of $x$ (the deviation of $a$ from $a_{0}$ ) versus $T$, (the cost of throttle activity). The Hamiltonian functional corresponding to the cost index is

$$
H(x, T)=\frac{1}{2}\left[q_{1} x^{2}+q_{2} T^{2}\right]-2 p \frac{x^{2}}{\mu}\left[\frac{\bar{T}_{\text {по }} \mid\left[V_{r} \sin \left(\theta+V_{v} \cos (\theta)\right)\right]}{m}\right] T
$$

\section{Equation 11}

Following procedures laid down by the Calculus of Variations the co-state equation is derived by evaluating

$$
\dot{p}=-\frac{\delta h}{\delta x}=-q_{1} x=4 p \frac{a_{0}}{v} \frac{\bar{T}_{\text {nот }}\left[V_{r} \sin \left(\theta+V_{v} \cos (\theta)\right)\right]}{m} T
$$

\section{Equation 12}

The variable $p$ is referred to as the co-state and serves as an adaptive gain factor in the control law. The unconstrained control equation, or the optimal control feedback if there were no constraints on the throttle setting, are given by

$$
\frac{\delta H}{\delta T}=0
$$

Equation 13

$$
T_{u}=-4 \frac{p}{q_{2}} \frac{a_{0}}{v} \times\left[\frac{T_{\text {nom }}\left(V_{r} \sin \theta+V_{v} \cos \theta\right)}{m}\right]
$$

\section{Equation 14}

\section{Constrained Control Law}

The optimal control derived in the previous paragraph assumes that the throttle $T$ can assume any numerical value.

The realizable throttle setting is constrained by minimum and maximum values, $T_{\min }$ and $T_{\max }$. Thus the optimal control law must be constrained accordingly for appropriate values of $T<T_{u}$ via the following method of determination.

$$
\begin{aligned}
& \text { if }\left(T_{u}>T_{\max }\right) \rightarrow T=T_{\max } \\
& \text { if }\left(T_{\min } \leq T_{u} \leq T_{\max }\right) \rightarrow T=T_{u} \\
& \text { if }\left(T_{\max } \leq T_{u}\right) \rightarrow T=0
\end{aligned}
$$

\section{Equation 15}

In Eq. (15), $T_{u}$ is the unconstrained command; and $T$ is the constrained command - the throttle position that is actually commanded by the regulator.

The constrained control law of Eq. (15) is sub-optimal for $T_{u}$ $<T_{\min }$ and $T_{u}>T_{\max }$. This sub-optimality can often lead to poor regulator performance, or worse yet, closed-loop system instability. However, with proper tuning of the weighting parameters $q_{1}$ and $q_{2}$, the throttle regulator has an acceptable level of performance. 


\section{N. Initial Condition for the Co-State}

Assuming that the regulator is initialized just after the initial de-orbit burn at apogee where the dissipative forces are nearly zero, the required initial throttle is approximately zero. Consequently, the co-state can be set as $p_{o}=0$ with no loss of generality in the problem. Because an initial value is specified for $p$, the co-state equation may be integrated forward in time instead of backward -- a practical limit of many optimal control laws.

\section{O. First Order Filter}

The actual throttle commands generated from the above control law were first filtered before commanding the rocket engine. A simple first order filter was implemented to reduce engine command jitters or commands that spiked frequently and would be less efficient to implement by smoothing and delaying response. A first order filter only has one parameter, $\tau$ or time constant, to change. Figure 9 illustrate how an unfiltered response compares to a less demanding filtered response.
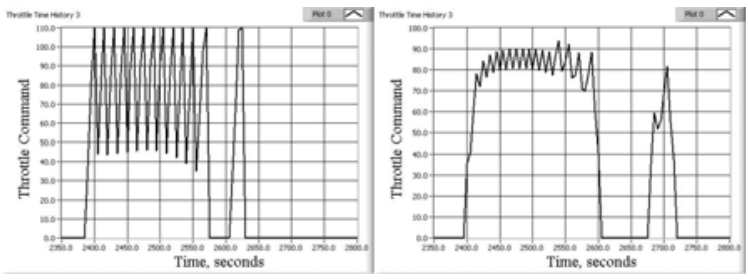

Figure 9- Throttle Time History; Results shown a) without filter, b) with filter applied

The filtered response allows for less severe requirements to be imposed on the engine. The areas under each of the curves are approximately equal because the vehicle still needs to overcome the same amount of energy lost to drag during the maneuver.

\section{P. Regulator Control Gain Selection}

As mentioned previously, the gain parameters in Eq. (10), $q_{1}$ and $q_{2}$, weight the relative cost of $x$ (the deviation of $a$ from $a_{0}$ ) versus $T$, (the cost of throttle activity). A larger $q_{1}$ value drives the regulator to match the current orbit's semi-major axis, $a$ to the original semi-major axis, $a_{0}$. More oscillations in throttle commands are generated with higher values of $q 1$ because the regulator tries harder to match the original orbital energy. A larger $q_{2}$ gain value introduces more fuel usage into each throttle input to help reduce throttle activity.

Several test runs of the aero-assist simulation were conducted to see how the vehicle's performance responded to changes in chosen gain values. An important aspect during testing was to realize that the individual magnitudes of the gain variables were not the key to regulator performance. Rather, $Q$ the ratio between $q_{2}$ and $q_{1}$ determined the performance level attained by the regulator. A Monte-Carlo type analysis was conducted to observe the effects of the gain ratio, $Q$, to regulator performance in helping the vehicle achieve the maximum inclination change possible.

\section{Q. Engine Performance}

A sample throttle time history is shown below in Fig. (10). These filtered throttle commands were given to the rocket engine during a simulated aero-assisted maneuver that took four orbits to complete. The thrust levels vary based on the drag experienced through the atmospheric pass and the necessary thrust for orbit stabilization. The four commands also require a level of engine throttling for a standard 3,300 lb nominal thrust engine. Rocket engines can be throttled in practice by reducing the propellant flow rate. This mechanism for throttling can be achieved through modulating the injector pressure.

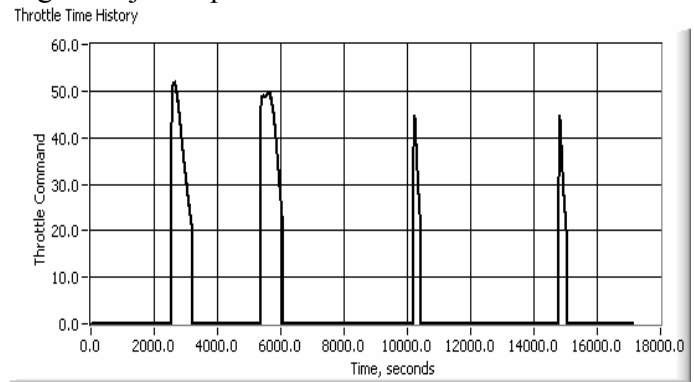

Figure 10- Throttle Time History

A separate simulation analysis tool was built to model a throttled liquid engine and its response capability. The focus of this simulation tool is in the transient start up and modeling the change over very small time steps in order to verify that the engine performance is not compromised when a throttle command is given. The results of the simulation receiving a $50 \%$ throttle command can be seen in the following plots shown in Fig. (11). Displayed below are several plots that show how the mass flow, injector pressure, thrust and stagnation pressure all change with the throttle command. As desired there is an immediate drop in thrust levels from the resulting drops in mass flow rate and consequently stagnation pressure from the given command. Also important to notice is that the pressure ratio between the injector and the combustor stays above 1.25.

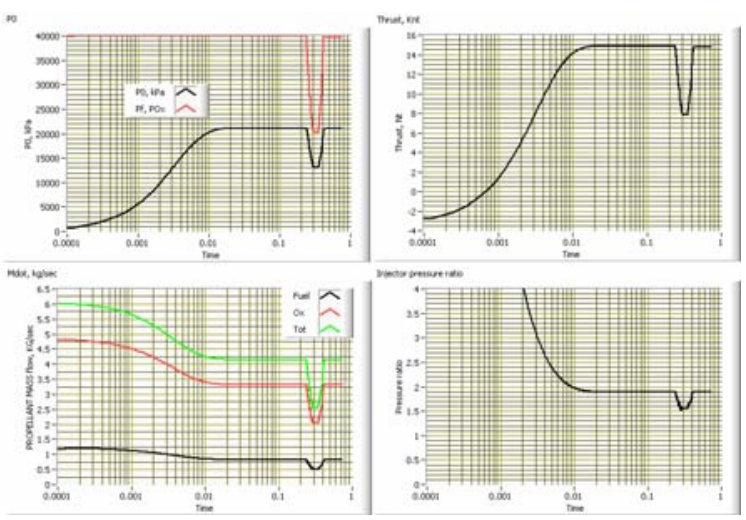

Figure 11- Results from $50 \%$ throttle command

The relationship between the commanded throttle, or desired thrust level, and the upstream injector pressure ratio are not 1:1. Figure 12 shows how near a desired thrust level of $25 \%$ the rocket engine begins to become increasingly unstable and performance quickly drops off as combustion instabilities begin. By examining the data presented in Fig. 12 it became apparent that the minimum throttle command that could be allowed is near $35 \%$. A throttle level of $35 \%$ ensures that the regulator pressure level stays above 1.25 and stays above the zone where combustion instability may occur. 


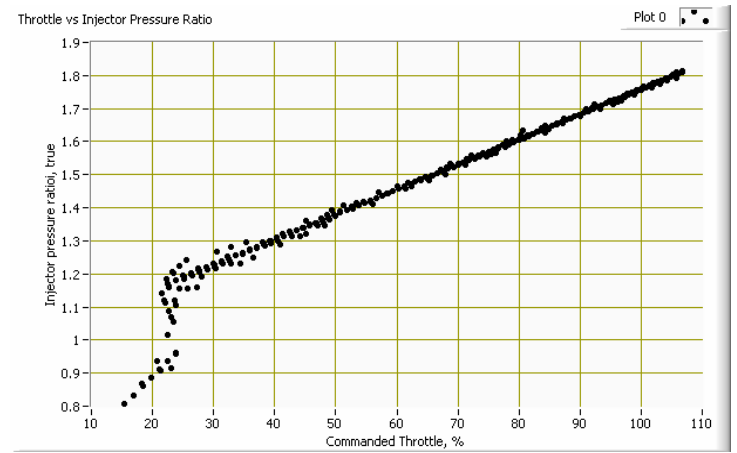

Figure 12- Throttle vs Injector Pressure Ratio

\section{HEATING}

This portion of the paper will investigate the ability of the vehicle to survive multiple re-entry scenarios and how it can effectively deal with the associated loads due to heating.

\section{R. Lumped-Mass Heating Approximation for Nose cap Skin and Leading Edge}

The heating calculations model the convective and radiative flow heating behind bow shock, back radiation from the nose cap surface to space, and heat absorption by the structure. The stagnation point heating calculations and models to be presented in this document are adapted from the method of Faye and Riddel $^{23}$ and implemented by Quinn and Gong ${ }^{24}$. This semiempirical method includes many real gas effects behind the bow shockwave including variable specific heat and species diffusion. Reacting gas chemistry and wall catalyticity are not modeled. The model is an "engineering" code; however, it does provide a good real-time tool for evaluating comparative heating and temperature levels for the various trajectories. The proximity of the vehicle nose stagnation point is present in Fig. (13). The highest heating levels to be experienced by the vehicle lie just behind the normal shock portion of the bow shockwave.

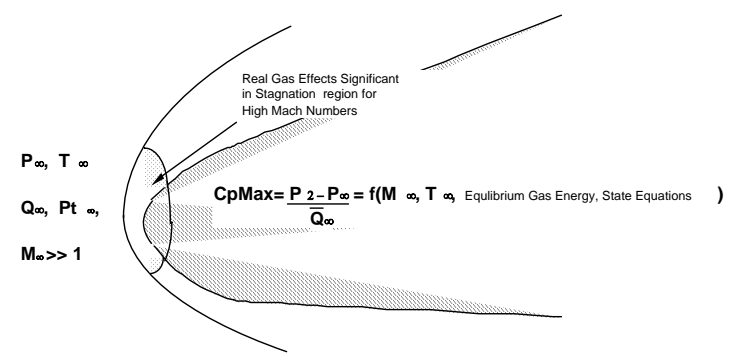

Figure 13- Schematic of Stagnation Point Region

The skin is assumed to be a thin monolithic slab of reinforced carbon-carbon (RCC) composite with a FRSI (Felt Reusable Surface Insulation) thermal protection system (TPS) backing. This FRSI backing is assumed to perfectly insulate the nose cap structure from the remainder of the spacecraft airframe. An ablative coating of Avcoat-5026-39 ${ }^{25,26}$, an epoxy-phenolic resin is superimposed over the nose cap wall to be considered in the design although is not desired. Issues with bonding of the two materials including differential expansion and insuring uniform recession rates are not considered here. Avcoat-5026-39 was chosen as a possible ablator because of its widely available data and is only used here as a baseline in determining the need of ablatives on the vehicle.

Because of the high thermal conductivity ( $\kappa)$ of the nose cap wall and the thinness of the wall when compared to the radius of the nose cap, the $\operatorname{Biot}^{27}$ number is small and the nose cap wall is effectively modeled as a "lumped-mass" structure with constant temperature throughout. The wall thickness is defined as the depth of the ablative layer and the RCC thickness and the average head capacity of the wall is the thickness-weighted heat capacity of the ablative and RCC wall layers. The "lumped-mass" temperature approximation for this wall segment can be written as

$$
\dot{q}_{\text {in }}-\dot{q}_{\text {out }}=\left[\rho_{\text {Avcoat }} C_{p_{\text {Avcoat }}} t_{\text {Avcoat }}+\rho_{R C C} C_{p_{R C C}} t_{R C C}\right] \dot{T}_{\text {wall }}
$$

\section{Equation 16}

In Eq. (16), $\dot{q}_{\text {in }}$ is the heat flux into the wall, $\dot{q}_{\text {out }}$ is the heat flux out of the wall, $\dot{T}_{\text {wall }}$ is the time rate of change of the wall temperature, and the term is brackets is the average heat capacity of the wall segment. Since the values of the specific heat and density are thermal properties of the material, the only way to significantly vary the heat capacity is to change the material thickness.

\section{Modeling the Heat Fluxes Into and Out of the Wall Segment}

There are two primary heat flux sources into the nose cap modeled in this analysis: 1) stagnation heating from high-speed external flow, and 2) radiative heating from the high-speed external flow. For the high Mach number conditions being modeled here, the effects of solar heating are considered negligible. There are also three primary heat flux sources out of the nose cap that are modeled in this analysis; 1) back radiation from nose-cap surface to the black background of space, 2) ablative cooling from the Avcoat layer coating the outer surface, and 3) circumferential heat conduction along the nose cap. While heat soak back through the FRSI blankets and nose cap attachment structures is a significant design problem for long duration flights; for the moderate duration analyses being modeled here, this effect is considered to be negligible. By ignoring thermal soak back in this analysis the predicted temperatures will be conservative; that is, the surface temperature levels to be experienced by the real vehicle will be less than predicted by this model.

\section{Stagnation Heat Flux into the Nose cap}

The stagnation heat flux is modeled as forced convection with the heat transfer coefficient modeled using the method of Faye and Riddel(Ref. 23).

The stagnation temperature behind the shock wave is evaluated using a linear interpolation of real-gas enthalpy and specific heat tables (Ref.16) for air. Since $\gamma$ is no longer constant across the shock wave an approximation is made by interpolating the tables of gas properties for air (Ref.16) using Eckert's reference temperature with the actual wall temperature replacing the adiabatic wall temperature.

The specific heat ratio based on the reference temperature approximation, the ideal gas normal shock equations (Ref. 13 above) are solved for the stagnation and static flow properties behind the shock wave. The process is iterative, but is typically solved in just a few cycles. Once the flow properties behind the shock wave are evaluated, the Fay and Riddel formula with no gas dissociation and a Prandtl number of 0.71 computes the heat transfer coefficient. The stagnation properties are evaluated based on the stagnation temperature behind the normal shock wave and the wall temperatures are evaluated using the wall temperatures. 


\section{Heating Relief Correction for Three-Dimensional Nose cap}

Away from the stagnation point, the amount of heating drops off rapidly. Anderson ${ }^{28}$ presents an empirical correction factor for this heating drop-off as a function of the surface incidence angle. The heating relief factor, $\Phi(\theta)$ is a function of the flow incidence angle. The parameter $\Phi(\theta)$ is the ratio of the local heating rate to the stagnation heating rate.

\section{External Flow Radiation Heating}

For hypersonic conditions the flow behind the normal shock wave becomes so hot that it begins re-radiating the energy.

\section{S. Leading Edge Heating Correction}

The leading edge of the waverider will be exposed the most to the harsh environment experienced during an aero-pass. Most of the heating generated from stagnation heating will be encountered along the leading edge. The leading edge also provides the best place to begin working a solution for active cooling. Because the leading edge introduces a three dimensional nose cap an averaged heating relief factor must be found using the methods already described above.

An option for modeling the effects of active cooling methods has been allowed for in this code. For this application a simple constant rate of heat-flux subtraction will be included. This parameter can be adjusted as need to keep the surface temperature within bounds, and then a detailed active cooling system can be designed to meet the required level of heat flux. This method is invaluable for analyzing the effectiveness of a cooled leading edge based on the length of leading edge used. An example of a leading edge only partially cooled along the front most edges is shown below in Fig. (14). The bulk of the stagnation heating load will be applied on the front most tip of the vehicle where the shock waves are formed. Analysis has shown that leading edge cooling is not necessary along the full length of the waverider vehicle. This means that the cooling system can be more compact and focus on cooling the immediate source of the majority of the heating found at the tip.

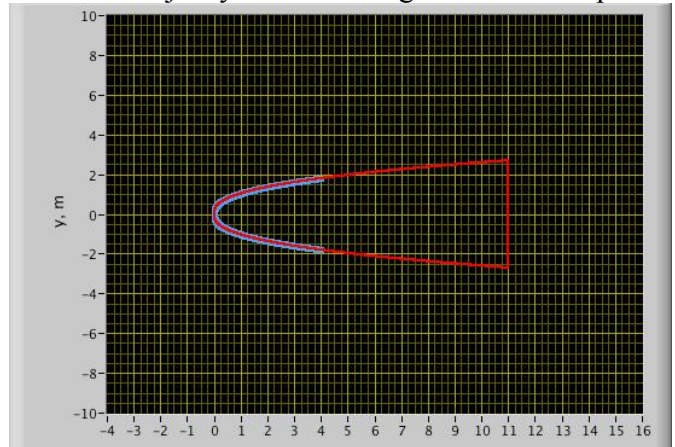

Figure 14- Variable Length Cooled Leading Edge

\section{T. Heating Results}

Fortunately, a new generation of thermal protection materials developed at NASA Ames Research Center ${ }^{29,30}$ may offer an additional solution to the heating problem. Heating would not be a concern if the waverider's leading edges were equipped with Ultra-High Temperature Ceramics (UHTC) ${ }^{31}$ material and the remainder of the nose cap was covered in reinforced carboncarbon (RCC) as analyzed above. Heating analysis has shown that without leading edge cooling the wall temperatures reach a maximum temperature of 3800 degrees Fahrenheit. The maximum limits for RCC is only 3000 degrees Fahrenheit before they begin to oxidize and ablate. Therefore, a transpiration cooled leading edge must be implemented or some other means of heat management must be employed to dissipate sufficient heat away for survival. Figure 15 shows a time history of wall temperature during an aero-assisted maneuver. The addition of UHTC material would allow this type of trajectory to be executed well within the survivability limits of the vehicle. Additionally, it can be seen from Fig. (15) that the duration of heating is very short. As soon as the vehicle exits the atmosphere it immediately begins re-radiating most of its heat back out to space. This allows for the vehicle to quickly cool before it is required to make another aero pass. Heating levels can also be modulated by how the aero pass is initiated. The more aggressive, more dynamic pressure loading experienced in a pass will increase the level of heating.

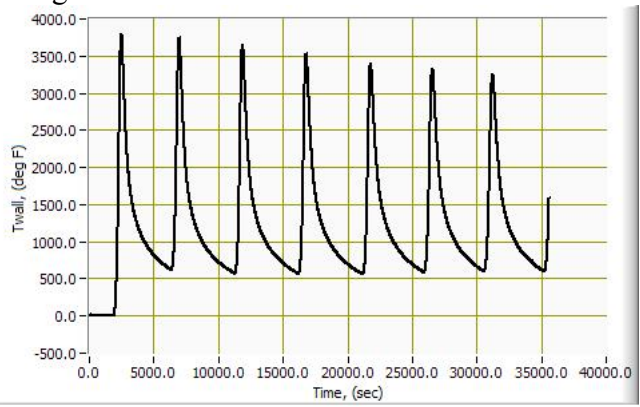

Figure 15- Wall Temperature Time History

\section{SIMULATING RESPONSIVE SPACE}

The focus of the paper up to this point has been to establish which technologies and capabilities are necessary in order to perform an aero-assist maneuver. The focus now shifts to what an aero-assist maneuver fully simulated would look like and the options available to such a spacecraft.

\section{U. Description of the 3-DOF Simulation Model}

This section presents the results of the 3-DOF simulation analyses. The study was facilitated by the development of an interactive real-time, "piloted" simulation with a graphical user interface (GUI). The interactive simulation allows for a rapid evaluation of a wide variety of candidate maneuvers and trajectories.

The simulation served to provide insight as to which parameters were critical to the problem, and which parameters could be effectively ignored. This was found to be very useful in this analysis. All trajectories presented in this section are not the result of optimization, but instead are "representative" trajectories that illustrate the capabilities and deficiencies of each of the different configurations being analyzed. Trajectories derived from this analysis are an excellent point of departure for more detailed and narrower-based configuration studies that employ formal optimization strategies ${ }^{32}$.

The differential equations used to describe the orbital motion of the spacecraft are derived using the satellite coordinate system $^{33}$. In this coordinate system, the r-component points away from the center of the Earth in a radial direction, the ncomponent is perpendicular to the radial direction and points in the direction of travel of the spacecraft, and the $i$-component completes the right-handed orthogonal coordinate system. This coordinate system stays fixed to the spacecraft at all times, and the $i$-coordinate is always perpendicular to the instantaneous orbital plane. Figure 23 depicts the forces acting on the spacecraft during the atmospheric encounter. Gravity $\left(F_{\text {grav }}\right)$ acts in the radial direction towards the center of the Earth, and aerodynamic drag and lift generated by the spacecraft act along and perpendicular to the direction of motion. The angle of motion 
relative to the local horizontal is the described by the flight path angle, $\gamma$. The direction of motion is along the orbital track and has an inclination angle $i$. The lift vector can be rolled transverse to the direction of motion via the bank angle, $\phi$. The position of the spacecraft relative to the orbital perigee is described by the true anomaly angle, $v$, and the radial position of the spacecraft relative to the center of the Earth is designated by the symbol, $r$. The symbols, $V_{r}$, and $\mathrm{V}_{v}$ designate the instantaneous vertical and horizontal velocity components. The inclination of the spacecraft longitudinal axis with respect to the instantaneous horizontal plane is designated by the symbol, $\theta$.

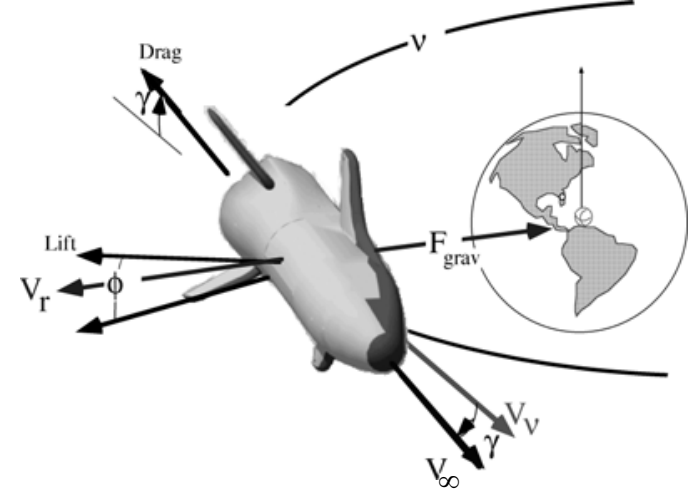

Figure 23- Forces Acting on a Spacecraft During Atmospheric Interface

The 3-DOF equations of motion can be written as a 6-state system of equations. Eq. (17) presents the non-linear set of equations in vector form. In the equations a term for propulsive thrust, $F_{\text {thrust }}$, has been allowed for generality. The system thrust is related to the change in vehicle mass, $m$, by the specific, $I_{s p}$, of the propulsive system. In this model a simple single harmonicgravity model is used. Additional undefined terms in Eq. (17) include, $\mu$, the gravitational planetary constant for the Earth, and $g_{0}$, the acceleration of gravity at sea level.

$\left[\begin{array}{c}\dot{V}_{r} \\ \dot{V}_{v} \\ (i) \\ \dot{r} \\ \dot{v} \\ \dot{m}\end{array}\right]=\left[\begin{array}{c}\frac{V_{v}{ }^{2}}{r}+\frac{F_{\text {lift }} \cos (\gamma) \cos (\phi)-F_{\text {drag }} \sin (\gamma)+F_{\text {thrus }} \sin (\theta)}{m}-\frac{\mu}{r^{2}} \\ -\left[\frac{V_{r} V_{v}}{r}+\frac{F_{l i f t} \sin (\gamma) \cos (\phi)+F_{\text {drag }} \cos (\gamma)-F_{\text {thrust }} \cos (\theta)}{m}\right] \\ -\frac{F_{l i f t} \sin (\phi)}{m V_{v}} \\ \\ V_{r} \\ \frac{V_{v}}{r} \\ -\frac{F_{\text {thrust }}}{g_{0} I_{s p}}\end{array}\right]$

Equation 17

The lift force and drag forces, $F_{\text {lift }}$ and $F_{\text {drag }}$ are calculated from a table lookup of the trim lift and drag coefficients as presented earlier in the Waverider Vehicle Aerodynamics section. The trim lift and drag coefficient tables implicitly bring the control surface deflections and longitudinal trim of the vehicle into the simulations. Transforming the velocity vector components from the satellite reference coordinate system to the Earth-fixed WGS-84 coordinate system ${ }^{34}$ and expressing in polar form calculates the angle-of-attack. For wings level flight the angle-of-attack can be approximated by $\alpha=\theta-\gamma$. Only trimmed angles-of-attack are allowed for the vehicle, and hold the last values used in the lookup tables when trimmed angles-of-attack are exceeded. The WGS- 84 transformations also calculate the geodetic latitude, $\lambda$, geocentric longitude, $\delta$, and geometric altitude, $h$, of the spacecraft. The atmospheric model can be selected to use either the 1977 US standard atmosphere or the 1999 Global Reference Atmospheric Model $^{35}$. The pitch and roll angle of the spacecraft are commanded by setting a nominal profile for a given flight trajectory and can also be modified in real-time by a "pilot" input using a video-game joystick. A variety of displays are available to the "pilot" to aid in flying the desired trajectories. Figure 16 shows a portion of the GUI front panel display for the simulation that was used to analyze the sample trajectory scenarios for aero-assist maneuvers starting in LEO.

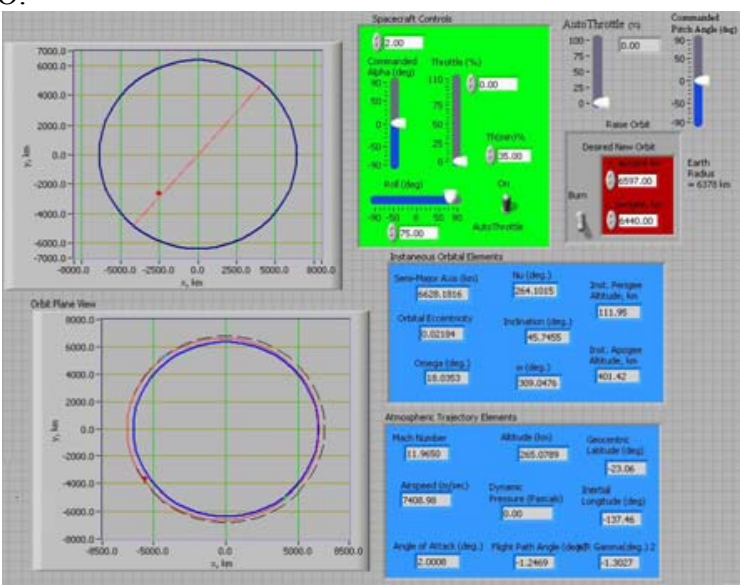

Figure 16- Aero-Assist Simulation GUI

\section{RESULTS AND CONCLUSIONS}

This section will examine the aero-assist scenarios for the waverider vehicle. A representative trajectory of an aero-assisted trajectory is presented below. Figures 17 and 18 illustrate how the arrival time over target is significantly changed when aeroassist is used to modify the original orbit.

Figure 18 shows that with appropriate aero-assist maneuvers a single ground target may be flown over on successive orbits with arrival times that are extremely unpredictable. The ability to rapidly vary orbital parameters makes it difficult for hostile forces to predict the footprint of orbiting platform. Such a platform deployed during a time of crisis would be invaluable as technical intelligence gathering option. It must be recognized that aggressive maneuvering also is accompanied by high heating rates and will required higher propellant usages. These issues certainly must be worked and resolved before an operational vehicle is deployed. 


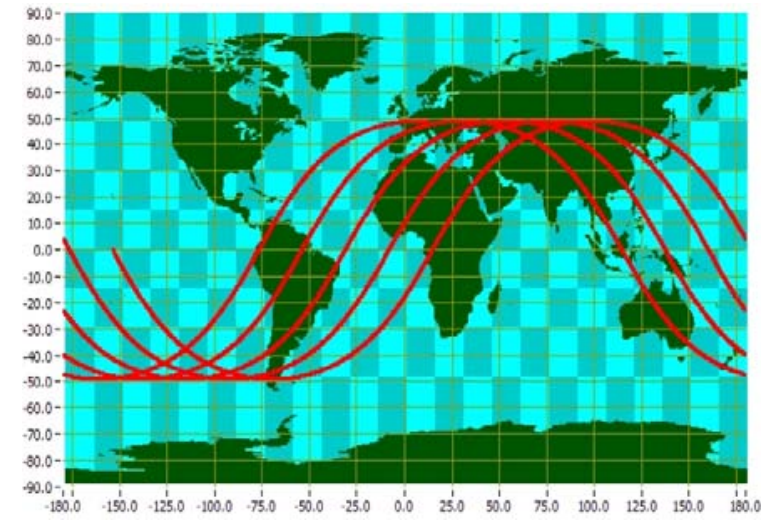

Figure 17- Example of a Fixed Orbit

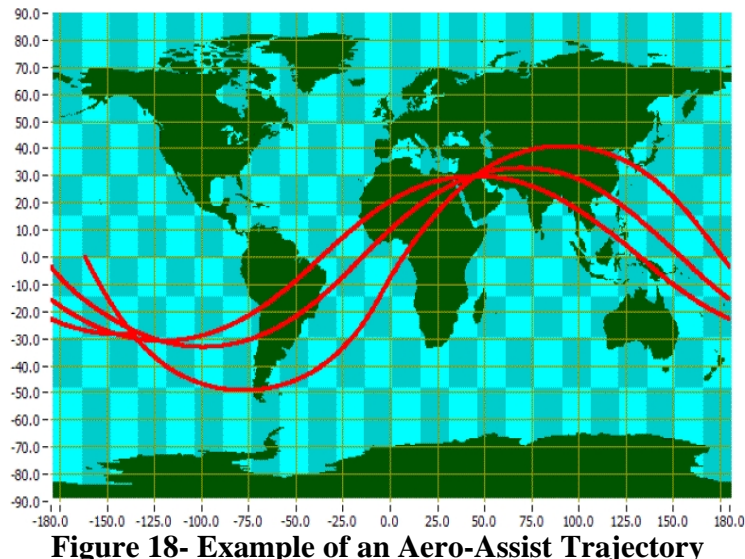

It is obvious from the above figures how the aero-assisted trajectory is beneficial for unpredictable reconnaissance. The period for a satellite in LEO is approximately 90 minutes which makes this entire operation occur in just a few hours. If a spacecraft were to perform an aero-assist maneuver like the ones presented above they would need nearly three times the amount

\section{REFERENCES}

1 Curtis, H. D., Orbital Mechanics for Engineering Students, Elsevier Butterworth-Heinemann, Burlington, MA, 2005.

${ }^{2}$ Rothman, J., and Siegenthaler, E., "Responsive Space Launch The F-15 Microsatellite Launch Vehicle," AIAA $1^{\text {st }}$ Responsive Space Conferece, Redondo Beach, CA, paper LA Section/SSTC 2003-9002

3 Walberg, G. D., "A Survey of Aeroassisted Orbit Transfer," AIAA $9^{\text {th }}$ Atmospheric Flight Mechanics Conference, San Diego, CA, public domain

${ }^{4}$ Anderson, J. D, Jr., Lewis, M. J., Kothari, A. P., and Corda, S., "Hypersonic Waveriders for Planetary Atmospheres," AIAA Journal of Spacecraft and Rockets, Vol. 28, No. 4, 1991, pp. 402.

Pienkowski, John P., Whitmore, Stephen A., and Spencer, Michael G., "Analysis of the Aerodynamic Orbital Transfer Capabilities of X-37 Space Maneuvering Vehicle (SMV), " AIAA paper \# 2003-0908, January 2003. paper presented at the 41st Aerospace Sciences Conference, Reno, NV, Jan 6-9, 2003.

${ }_{6}$ Dunbar, Bonnie J., Whitmore, Stephen A., “Orbital Space Plane: Past, Present, and Future" AIAA paper \#20032718, July 2003 of fuel. Figure 19 illustrates the difference in an aero-assist to all-propulsive plane change maneuver.

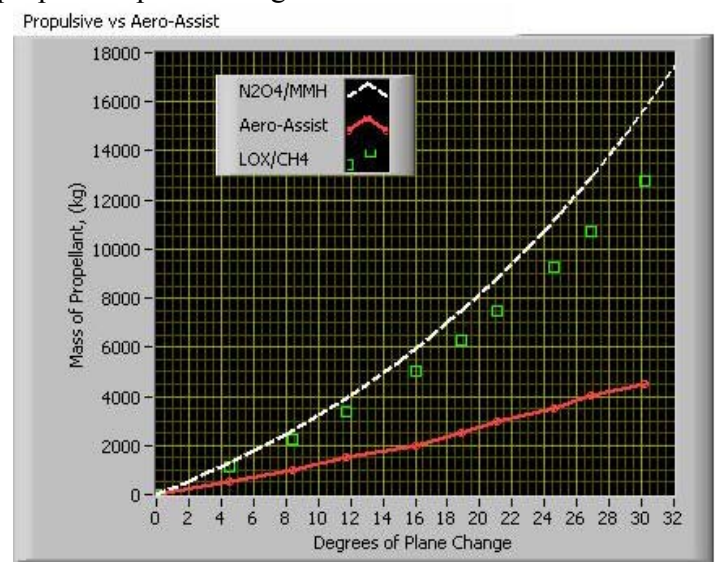

Figure 19- Comparison of Fuel Expenditure

The ability for an orbiting reconnaissance platform to rapidly vary its orbital parameters would make it difficult for hostile forces to predict the coverage time over target. This capability has been long sought after by the Department of Defense. Additionally, the ability to perform an aero-assist maneuver would allow two missions to be conducted in space for the price of one launch. For example, NASA cannot currently service the Hubble Telescope and the International Space Station because they are in two different orbit inclinations. The advantages of aero-assist will continue to manifest themselves; however, they will not be realized without a vehicle capable of executing the required propulsive and aerodynamic maneuvers. This paper has sought to demonstrate that the waverider vehicle with UHTC material leading edges and equipped with a throttled engine and a high hypersonic L/D ratio and flight control surfaces will be able to perform an aero-assist maneuver. Simulation tools were created to analyze each piece of the puzzle so that this systems level approach could cover each of the needed requirements to fulfill flight worthiness.

7 Anderson, J. D. Jr., Modern Compressible Flow with Historical Perspective, $3^{\text {rd }}$ ed. McGraw Hill Higher Education, New York, 2003, Chap. 4

8 Anderson, J.D. Jr,, Ferguson, F., and Lewis, M.J., "Hypersonic Waveriders for High Altitude Applications," AIAA paper \# 91-0530, January 1991. paper presented at the $29^{\text {th }}$ Aerospace Sciences Conference, Reno, NV, Jan 7-10, 1991.

9 Bowcutt, K.G., Anderson, J.D., Jr., and Capriotti, D.P., "Viscous Optimized Hypersonic Waveriders," AIAA paper \# 87-0272, presented at the AIAA 25 ${ }^{\text {th }}$ Aerospace Sciences Meeting, Reno, NV, Jan. 12-15, 1987.

10 Sims, J. A., Longuski, J. M., and Patel, M. R., "Aerogravity-Assist Trajectories to the Outer Planets and the Effect of Drag," AIAA Journal of Spacecraft and Rockets, Vol. 37, No. 1, Jan. 2000, pp., 49-55.

11 Randolph, J.E., Davis, M.R., Leavens, T.J., Miyake, R.N., "A Waverider Application of an Advanced Nuclear Power Source."

${ }^{12}$ Anderson, J. D. Jr., Hypersonic And High Temperature Gas Dynamics, American Institute of Aeronautics and Astronautics, Inc., Reston, VA, 1989, Chapter 3.

${ }_{13}$ Anderson, J. D. Jr., Modern Compressible Flow with Historical Perspective, $3^{\text {rd }}$. ed., McGraw-Hill Higher Education, Hew York, 2003, Chapter 10. 
14 Whitmore, S. A., "Conical Supersonic Flow Fields," URL:

http://www.engineering.usu.edu/classes/mae/5420/Compre ssible fluids/section11.html, [ cited 15 November 2005].

${ }^{15}$ Mills, Anthony F., Heat Transfer, Irwin Publishing, Homewood, IL, 1992, Chapter 4.

16 Hansen, C. Frederick, "Approximations for the Thermodynamic and Transport Properties of HighTemperature Air," NASA TR R-50, 1959.

${ }^{17}$ Motyard, E., J., and Loposbe, J., D., Average SkinFriction Drag Coefficients From tank Tests of a Parabolic Body of Revolution, NACA TN-2854, Washington, D.C, 1953.

${ }^{18}$ Von Karman, T., Turbulence and Skin Friction, Journal of Aeronautical Sciences, Vol. 1, No 1, January 1934, pp. 120.

${ }^{19}$ Carlson, John R., "Prediction of Very High Reynolds Number Compressible Skin Friction," AIAA Paper-98-2880, 1998.

20 Sommer, S.C., and Short, B.J., "Free-Flight Measurements of Turbulent Boundary-Layer Skin Friction in the Presence of Severe Aerodynamic Heating at Mach Numbers From 2.8 to 7.0," NACA TN-3391, March, 1955.

${ }^{21}$ McBride, B. J., Gordon, S. "Chemical Equilibrium with Applications,"

URL:

http://www.grc.nasa.gov/WWW/CEAWeb/ [ cited [15 August 2006].

22 Kirk, Donald E., Optimal Control Theory: An Introduction, New Jersey: Prentice-Hall Inc., 1970.

${ }^{23}$ Fay, J. A. and F. R. Riddell, "Theory of Stagnation Point Heat Transfer in Dissociated Air," Journal of the Aeronautical Sciences, vol. 25, no. 2, February 1958, pp. 73-85, 121.

${ }^{24}$ Quinn, Robert D. and Leslie Gong, A Method for Calculating Transient Surface Temperatures and Surface Heating Rates for High-Speed Aircraft, NASA/TP-2000209034, December, 2000.

${ }^{25}$ Crouch, R.K., and Walberg, G.D., "An Investigation of Ablation Behavior of Avcoat 5026/39M Over a Wide Range of Thermal Environments”, NASA TM X-1778, 1969.

${ }^{26}$ Cleland, J., and Iannetti, F., " Thermal Protection System of the Space Shuttle," NASA Contractor Report 4227, Contract NASW-3841-72, June 1989.

${ }^{27}$ White, F. M., Heat and Mass Transfer, Addison-Wesley Publishing Company, Reading MA, 1988, Ch 4.

${ }^{28}$ Anderson, John D., "Hypersonic and High Temperature Gas Dynamics," McGraw-Hill Inc., New York, 1989, Chapter 6.

${ }^{29}$ Florence, D. E., Fischer, G., Moderate Lift-to-Drag Aeroassist, NASA Report N85-16989 08-15, Lewis Research Center OTV Propulsion Issues, April 1984, pp. 69-84

30 Carpenter, Russell, "Current Systems Analysis: AEROASSIST FLIGHT EXPERIMENT", Lecture Notes for ASE 396, University of Texas at Austin, November 2, 1992 , [http://www.tsgc.utexas.edu/archive/fulltext/AeroassistFlightE xp.pdf]

31 Bull, J., Keese, D., Rasky, D., and Salute, J., "SHARPB2 Flight Test Objectives, Project Implementation and Initial Results," Proceedings from $2^{\text {nd }}$ Annual Conference on Composites, Materials and Structures, Cocoa Beach, FL, 22-25 Jan. 2001

${ }^{32}$ Whitmore, Stephen A., Banks, Daniel W., Andersen, Benjamin A., and Jolley, Patrick R., "Direct-Entry, Aerobraking, and Lifting Aerocapture for Human-Rated Lunar Return Vehicles,” AIAA paper \#2006-1033, January 2006.
33 Vallado, D. A. Fundamentals of Astrodynamics and Applications, 2nd ed. El Segundo, CA: Microcosm Press and Boston, MA: Kluwer Academic Publishers, 2001, Chapter 4.

${ }^{34}$ Haering, E. A., Jr., and Whitmore, S., A., FORTRAN Program for the Analysis of Ground Based Range Tracking Data--Usage and Derivations, NASA TM 104201, 1992.

35 Justus, C., G., ed. "GLOBAL REFERENCE ATMOSPHERIC MODEL (GRAM-99)", NASA Space Environments and Effects Program, URL: http://see.msfc.nasa.gov/tte/model_gram.htm, [cited: 29 November 2005]. 